\title{
A new culture-based method for rapid identification of microorganisms in polymicrobial blood cultures by MALDI-TOF MS
}

Walter Florio, Susanna Cappellini, Cesira Giordano, Alessandra Vecchione, Emilia Ghelardi and Antonella Lupetti (1)

\begin{abstract}
Background: The application of matrix-assisted laser desorption/ionization time of flight (MALDI-TOF) mass spectrometry (MS) to microbial identification has allowed the development of rapid methods for identification of microorganisms directly in positive, blood cultures (BCs). These methods can yield accurate results for monomicrobial BCs, but often fail to identify multiple microorganisms in polymicrobial BCs. The present study was aimed at establishing a rapid and simple method for identification of bacteria and yeast in polymicrobial BCs from patients with bloodstream infection.
\end{abstract}

Results: The rapid method herein proposed is based on short-term culture in liquid media allowing selective growth of microorganisms recovered from polymicrobial BCs, followed by rapid identification by MALDI-TOF MS. To evaluate the accuracy of this method, 56 polymicrobial BCs were comparatively analyzed with the rapid and routine methods. The results showed concordant identification for both microbial species in 43/50 (86\%) BCs containing two different microorganisms, and for two microbial species in six BCs containing more than two different species. Overall, 102/119 (85.7\%) microorganisms were concordantly identified by the rapid and routine methods using a cut-off value of 1.700 for valid identification. The mean time to identification after BC positivity was about $4.2 \mathrm{~h}$ for streptococci/enterococci, $8.7 \mathrm{~h}$ for staphylococci, $11.1 \mathrm{~h}$ for Gram-negative bacteria, and $14.4 \mathrm{~h}$ for yeast, allowing a significant time saving compared to the routine method.

Conclusions: The proposed method allowed rapid and reliable microbial identification in polymicrobial BCs, and could provide clinicians with timely, useful information to streamline empirical antimicrobial therapy in critically ill patients.

Keywords: Bloodstream infection, Polymicrobial blood culture, MALDI-TOF MS, Rapid identification, Selective media

\section{Background}

Bloodstream infections represent a major public health problem being a significant cause of morbidity and mortality worldwide [1-5]. Polymicrobial bloodstream infection is particularly severe, and is commonly associated with dramatically high mortality rates [6-8]. Blood culture (BC), the "gold standard" for the diagnosis of bloodstream infection, requires at least $48-72 \mathrm{~h}$ before the results of microbial identification and antimicrobial

\footnotetext{
* Correspondence: antonella.lupetti@med.unipi.it

Dipartimento di Ricerca Traslazionale e delle Nuove Tecnologie in Medicina e Chirurgia, Università di Pisa, Via San Zeno 37, 56127 Pisa, Italy
}

susceptibility testing can be reported to clinicians. Time to identification may take an additional $18-24 \mathrm{~h}$ for polymicrobial $\mathrm{BCs}$ as compared to monomicrobial $\mathrm{BCs}$, since sub-culture onto the appropriate solid media may be necessary to isolate all microbial species. To reduce mortality, it is of vital importance to shorten time to diagnosis, and provide clinicians with the necessary information to promptly administer an effective antimicrobial therapy.

The development of new tools and techniques have allowed to reduce time to identification significantly as compared to culture-based methods. In particular,

(c) The Author(s). 2019 Open Access This article is distributed under the terms of the Creative Commons Attribution 4.0 International License (http://creativecommons.org/licenses/by/4.0/), which permits unrestricted use, distribution, and 
matrix-assisted laser desorption/ionization-time of flight (MALDI-TOF) mass spectrometry (MS) has proved a rapid and reliable method for microbial identification, and a number of recent studies indicate that it could be directly applied to identify microbial pathogens recovered from positive BCs [9-13]. However, microbial identification by MALDI-TOF MS directly in positive $\mathrm{BC}$ is intrinsically difficult for polymicrobial $\mathrm{BCs}$, since the peaks of multiple species merge into a single mass spectrum and the results obtained often yield the identification of the predominant microorganism only [14-20].

Direct microbial identification in polymicrobial BCs has proven problematic also when using automated microarray-based assays [21-23].

The aim of the present study was to establish a rapid method for MALDI-TOF MS microbial identification in polymicrobial BCs based on short-term sub-culture in liquid media allowing selective growth of individual species.

\section{Results}

Evaluation of the accuracy of a rapid method for identification of bacteria and yeast in polymicrobial blood cultures

Overall, 2456 BCs were analyzed, of which 2324 (94.6\%) resulted monomicrobial, while $132(5.4 \%)$ revealed polymicrobial growth after Gram staining; among these latter, $56(42.4 \%)$ BCs were analyzed with both the rapid and routine methods since they met the inclusion criteria used in this study, i.e. from each patient, only the first positive $\mathrm{BC}$ containing two or more different categories of microorganisms. From the 56 selected BCs, a total of 119 microbial strains were isolated and identified by the routine method; of these, 102 were bacteria, and 17 yeast. In $50 \mathrm{BCs}$, two distinct microorganisms were identified; five $\mathrm{BCs}$ contained three different microbial species, and one $\mathrm{BC}$ revealed the presence of four species.

Among the bacterial isolates, 66 were Gram-positive cocci and 36 Gram-negative bacilli. The Gram-positive cocci included: thirty-two coagulase-negative staphylococci (14 Staphylococcus epidermidis, ten Staphylococcus haemolyticus, three Staphylococcus hominis, two Staphylococcus capitis, one Staphylococcus cohnii, one Staphylococcus pettenkoferi, one Staphylococcus warneri), two Staphylococcus aureus, 26 enterococci (18 Enterococcus faecalis, seven Enterococcus faecium, one Enterococcus raffinosus), four streptococci (three Streptococcus oralis, one Streptococcus pneumoniae), and two Granulicatella adiacens.

The Gram-negative bacilli included 16 Enterobacteriaceae (four Escherichia coli, four Klebsiella pneumoniae, three Serratia marcescens, one Serratia liquefaciens, one
Serratia urealytica, one Citrobacter koseri, one Proteus mirabilis, one member of the Enterobacter cloacae complex), and 20 non-fermenting bacteria (12 Pseudomonas aeruginosa, six Acinetobacter baumannii, one Stenotrophomonas maltophilia, one Burkholderia cepacia). The identified yeast species included 10 Candida parapsilosis and seven Candida albicans strains.

In 43 of the $50(86.0 \%)$ BCs in which two microorganisms were present, both were concordantly identified by the rapid and routine methods whereas in the remaining seven BCs (14.0\%), only one of the two microorganisms was concordantly identified. In these latter $\mathrm{BCs}$, the second microorganism was not identified by the rapid method (Table 1).

In the six BCs containing three or more microorganisms, two microbes were concordantly identified by the two methods, as reported in Table 1.

In the overall, 105 of the 119 (88.2\%) microorganisms were concordantly identified by the rapid and routine methods. In particular, all the $17(100 \%)$ yeasts and 88 out of 102 bacteria (86.3\%) were concordantly identified. Among bacteria, Gram-negative bacteria showed an identification rate of $94.4 \%$, enterococci/streptococci $87.1 \%$, and staphylococci $77.1 \%$ (Table 2). Among the microorganisms concordantly identified by the two methods, 83/105 (79.0\%) had a score $\geq 2.000,19 / 105$ (18.1\%) showed a score between 1.700 and 1.999 and only three $(2.9 \%)$ a score $<1.700$, all the latter three being yeast (Table 1). Considering the high concordance in the identification results obtained, the score threshold for valid species identification by the rapid method could be lowered down to 1.700. Adopting a cut-off value of 2.000, 83/119 (69.7\%) microorganisms were identified by the rapid method, whereas lowering the cut-off for valid identification down to $1.700,102 / 119$ (85.7\%) microorganisms could be identified.

\section{Time to identification by the rapid method}

The mean growth time in selective liquid media to reach a density of 0.5 McFarland was $10.6 \mathrm{~h}(4.6-20.7 \mathrm{~h})$ in Brilliant Green Bile Broth, $8.2 \mathrm{~h}(4.9-16.8 \mathrm{~h})$ in $\mathrm{m}$ Staphylococcus Broth, $3.7 \mathrm{~h}(1.1-22.4 \mathrm{~h})$ in Todd Hewitt Broth w Colistin/Nalidixic acid, and 13.6 h (8.6-20.6 h) in HB\&L Sabouraud (Table 2). The longest growth times were observed for $C$. parapsilosis $(20.6 \mathrm{~h})$ for yeast, $C$. koseri $(20.7 \mathrm{~h})$ for Gram-negative bacteria, S. capitis (16.8 h) for staphylococci, and $S$. pneumoniae $(22.4 \mathrm{~h})$ for streptococci/enterococci; the shortest growth times in the different groups of microorganisms were found for C. parapsilosis $(8.6 \mathrm{~h})$, E. coli $(4.6 \mathrm{~h})$, S. haemolyticus (4.9 h), and E. faecalis (1.1 h) (data not shown).

After incubation in selective media, cultures were still polymicrobial but enriched of the microbial species positively selected by the growth conditions. In most cases, 
Table 1 Identification score of microbial species identified by MALDI-TOF MS after growth in selective liquid media

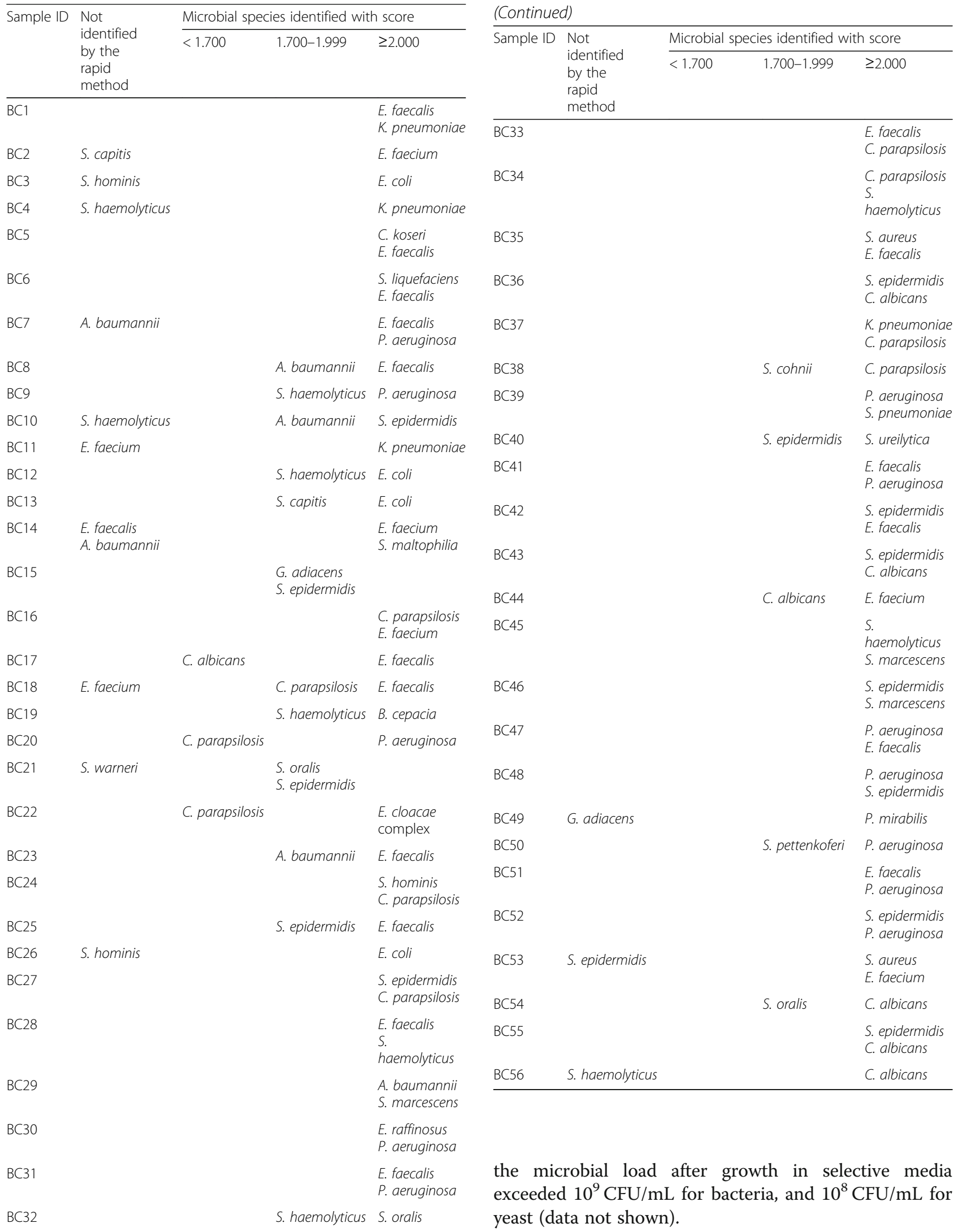

Table 1 Identification score of microbial species identified by MALDI-TOF MS after growth in selective liquid media yeast (data not shown). 
Table 2 Growth time in selective liquid media, time to identification after blood culture positivity, and number of concordant/ correct identifications by the rapid method

\begin{tabular}{|c|c|c|c|}
\hline $\begin{array}{l}\text { Selective culture medium } \\
\text { (selected category of microorganisms) }\end{array}$ & $\begin{array}{l}\text { Mean growth time to reach a density } \\
\text { of } 0.5 \text { McFarland (min-max) }\end{array}$ & $\begin{array}{l}\text { Mean time to identification } \\
\text { after BC positivity (min-max) }\end{array}$ & $\begin{array}{l}\text { Number of correct/ } \\
\text { concordant identifications (\%) }\end{array}$ \\
\hline $\begin{array}{l}\text { Brilliant Green Bile Broth } \\
\text { (Gram-negative bacteria) }\end{array}$ & $10.6 \mathrm{~h}(4.6-20.7 \mathrm{~h})$ & $11.1 \mathrm{~h}(5.1-21.2 \mathrm{~h})$ & $34 / 36(94.4 \%)$ \\
\hline m-Staphylococcus Broth (staphylococci) & $8.2 \mathrm{~h}(4.9-16.8 \mathrm{~h})$ & $8.7 \mathrm{~h}(5.4-17.3 \mathrm{~h})$ & $27 / 35(77.1 \%)$ \\
\hline $\begin{array}{l}\text { Todd Hewitt Broth w Colistin/Nalidixic acid } \\
\text { (streptococci/enterococci) }\end{array}$ & $3.7 \mathrm{~h}(1.1-22.4 \mathrm{~h})$ & $4.2 \mathrm{~h}(1.6-22.9 \mathrm{~h})$ & 27/31 (87.1\%) \\
\hline HB\&L Sabouraud (yeast) & $13.6 \mathrm{~h}(8.6-20.6 h)$ & $14.4 \mathrm{~h}(9.4-21.4 \mathrm{~h})$ & 17/17 (100\%) \\
\hline
\end{tabular}

Besides the incubation time in the selective media, the total identification time included about $20 \mathrm{~min}$ to recover, wash and resuspend bacteria and/or yeast, and 10 min, approximately, for MALDI-TOF MS analysis, for a total of about $30 \mathrm{~min}$. For identification of yeast, sample processing was about $15 \mathrm{~min}$ longer than for bacteria due to the necessity of an additional protein extraction step. The mean time to identification after $\mathrm{BC}$ positivity by the rapid method for the different microbial categories is reported in Table 2.

\section{Discussion}

Rapid identification of microbial pathogens by MALDITOF MS directly from positive BCs is inherently difficult for polymicrobial BCs. In many instances, one of the species is more abundant in the sample biomass, and MALDI-TOF MS analysis yields a single identification confidence score for the predominant microorganism [14-20]. In addition, increased error rates have been documented for polymicrobial in comparison to monomicrobial BCs in the context of direct MALDI-TOF MS [24]. Only a few studies reported that it may be possible to identify multiple microbial species in polymicrobial BCs by MALDI-TOF MS [16, 19, 25, 26]; however, the correct identification of the two different microorganisms was generally limited to a relatively low percentage of polymicrobial cultures $[16,19,26]$. In a recent study, the performance of the Bruker MBT Sepsityper IVD module was evaluated for the direct identification of microorganisms in polymicrobial BCs by MALDI-TOF MS [26]. Using this method, in more than $50 \%$ of polymicrobial BCs containing two species, the module correctly identified at least one of the different microorganisms, and in 49/143 (34.3\%) samples both microorganisms were identified. A confidence score value threshold $\geq 1.8$ was adopted for species identification, and the module's ability to detect multiple strains was limited to the identification of maximum two microorganisms.

Impaired performance for the identification of microbial pathogens in polymicrobial BCs have been reported also for molecular methods, such as the Accelerate Pheno system (Accelerate Diagnostics, Inc., Tucson, AZ, USA) [27], the Biofire FilmArray (BioFire Diagnostics,
Salt Lake City, UT) [28], QuickFISH (AdvanDx, Wolburn, MA) [15], and Verigene (Nanosphere, Northbrook, IL) $[15,28,29]$ molecular assays, although in most cases the number of polymicrobial BCs analyzed were very limited; therefore, the accuracy of the analyzed methods could not be determined precisely. Among the tested molecular methods, the best performance has been reported in a study combining the use of the MALDI BioTyper and FilmArray BCID panel compared to the culture-based method [30]. In that study, the MALDI BioTyper allowed to detect 31 polymicrobial BCs for which different Gram stain morphologies were not observed and, in the overall, the FilmArray BCID panel achieved complete identification in 153 (89.5\%) out of 171 polymicrobial BCs [30].

The method proposed in the present study is based on short-term culture in selective liquid media of microorganisms recovered from $\mathrm{BCs}$ resulting polymicrobial at the Gram stain, followed by MALDI-TOF MS identification. Using this method, in 43 (86\%) out of the 50 BCs where two microorganisms were present, both species were concordantly identified by the rapid and routine methods, and in all six BCs containing three or more microbial species, two of them were concordantly identified. In many instances, the lack of identification was due to the presence of two or more microorganisms able to grow in the same selective liquid medium. For example, in sample BC10, S. haemolyticus was not identified since its microbial load was much lower than that of S. epidermidis, simultaneously present. Analogous observations can be made with regard to samples $\mathrm{BC} 14$, BC18, BC21 and BC53. In sample BC49 the lack of identification of $G$. adiacens, concomitantly present together with $P$. mirabilis, might be due to the intrinsic resistance of Proteus spp. to colistin, thus resulting in its predominant growth.

Overall, using a cut-off of 2.000, 83/119 (69.7\%) microorganisms in polymicrobial BCs were concordantly identified by the rapid method. Adopting a cut-off of 1.700 concordant identification was obtained for 102/ 119 (85.7\%) microorganisms. Of note, identification was correct also for three yeast isolates identified with score values <1.700. Although score values $\geq 2.000$ are 
generally indicated by the systems' manufacturers for reliable species identification, it should be considered that this cut-off value has been established for MALDI-TOF MS identification of microorganisms in optimal conditions, i.e. from isolated colonies. However, in some instances, lowering the cut-off value may allow for rapid and correct microbial identification from samples resulting sub-optimal for MALDI-TOF MS analysis. For example, several authors [31-34] observed that cut-off values could be lowered down to some extent without compromising accuracy for MALDI-TOF MS identification of microorganisms recovered from positive BCs, which possibly contain residual blood components affecting the quality of spectra. In the present study, the quality of spectra and, hence, identification scores might be affected by the presence in the sample for MALDITOF MS analysis of residual blood components deriving from positive BCs and/or microbial cell material of counter-selected microorganisms after enrichment of the selected microbial species in liquid media. Indeed, lowering the cut-off value for species identification from 2.0 to 1.7 allowed to increase the concordant/correct identification rate of selectively grown microorganisms from 69.7 to $85.7 \%$ without affecting accuracy.

The main limitations of this rapid method are represented by i) the exclusion of polymicrobial BCs containing different microbial species that cannot be distinguished by Gram staining, and ii) the lack of identification of different microorganisms able to grow in the same selective medium. Conversely, the strength of the proposed method is that i) time to identification could be remarkably reduced compared to the routine method, which most often requires time consuming sub-culture for isolation of single species onto solid media, ii) it does not require specifically trained personnel, and iii) it is relatively inexpensive.

Despite the observed limitations of the proposed method, it should be considered that in $76.7 \%$ of the polymicrobial $\mathrm{BCs}$ analyzed, all the microorganisms present were identified by the rapid method.

In recent years, new methods have been proposed to achieve rapid antimicrobial susceptibility testing of bacteria and yeast by MALDI-TOF MS [35-37]. In the next future, further development of antimicrobial susceptibility testing by MALDI-TOF MS-based methods combined with the method herein described could provide relevant information on susceptibility/resistance of microbial pathogens from patients with polymicrobial infection with considerable time saving compared to the routine method.

\section{Conclusions}

In conclusion, the rapid MALDI-TOF MS identification method, herein described, is based on short-term culture in selective liquid media of microorganisms recovered from blood cultures that resulted polymicrobial after Gram staining. This rapid and simple method yielded concordant identification with the routine method by MALDI-TOF MS of both microorganisms present in 43 (86\%) out of 50 blood cultures containing two microorganisms, thus providing identification results at least 24 $h$ earlier than the routine method. Although the antimicrobial susceptibility testing cannot be shortened by this method, rapid identification of the infectious agents causing polymicrobial bloodstream infection could be helpful for the clinician to establish an effective empirical antimicrobial therapy for patients who are in particularly critical conditions.

\section{Methods}

\section{Study population}

Blood samples were drawn from patients recovered at the Cisanello Hospital of Pisa (Pisa, Italy). Blood cultures were processed at the Microbiology Unit of the Azienda Ospedaliero-Universitaria Pisana (Pisa, Italy). Samples were inoculated in both aerobic and anaerobic $\mathrm{BC}$ bottles (BACTEC Plus/F Aerobic, BACTEC Plus/F Anaerobic, BACTEC Peds Plus/F), and BCs were incubated in the BD BACTEC FX system (Becton Dickinson and Company; Milano, Italia) at $35+/-1.5^{\circ} \mathrm{C}$ for up to 5 days. From each patient, only the first $\mathrm{BC}$ resulting polymicrobial after Gram staining was included in the present study. These included: BCs positive for both Gram-positive and Gram-negative bacteria; BCs containing Gram-positive cocci both in clusters (staphylococci) and in chains (streptococci/enterococci), and BCs in which both Gram-positive and/or Gram-negative bacteria and yeast were detected.

Each BC was analysed using both the routine method and the rapid method established in the present study.

\section{Routine method for identification of microrganisms in polymicrobial BCs}

An aliquot of positive $\mathrm{BC}$ resulting polymicrobial at the Gram staining was plated onto appropriate solid media (blood agar, chocolate agar, MacConkey agar, Chapman agar, Schaedler agar for anaerobic cultures only and Sabouraud-dextrose agar for aerobic bottles only). Plates were incubated at $37^{\circ} \mathrm{C}, 5 \% \mathrm{CO}_{2}$ for $24-48 \mathrm{~h}$. Then, sub-cultures were streaked onto appropriate solid media, and microbial isolates were identified using a MALDITOF Microflex LT Mass Spectrometer (Bruker Daltonics) equipped with a MALDI Biotyper 3.0 software (Bruker Daltonics).

Samples were spotted onto the target plate, and overlaid with $1 \mu \mathrm{L}$ alpha-cyano-4-hydroxycinnamic acid (HCCA) matrix before MALDI-TOF MS analysis. 
Rapid method for identification of bacteria and yeast from polymicrobial BCs

An 8-mL aliquot of $\mathrm{BC}$ resulting polymicrobial after Gram staining was transferred into a BD Serum Separator Tube (BD Vacutainer System, Becton Dickinson). After centrifugation at $2000 \times \mathrm{g}$ for $10 \mathrm{~min}$, bacteria/yeast were collected from the surface of the silicon layer of the Serum Separator Tube, and suspended in 0.9\% saline at final density of $0.65 \mathrm{McF}$ arland (DensiCHECK plus, bioMérieux, l'Etoile, France). A $200-\mu \mathrm{L}$ aliquot of microbial suspension was used to inoculate the selective broths described below. Serial dilutions of the microbial suspension, in $0.9 \%$ saline, were plated onto TSH blood agar (bioMérieux), and the number of colony forming units (CFU) per $\mathrm{mL}$ of bacterial suspension was assessed by counting the number of colonies grown on plates after $24 \mathrm{~h}$ incubation at $37^{\circ} \mathrm{C}$.

The following selective liquid media were used: Brilliant Green Bile Broth 2\% (Biolife Italiana Mascia Brunelli; Milano, Italia), for selective growth of Gramnegative bacteria; Todd Hewitt Broth w Colistin/Nalidixic Acid (Liofilchem; Roseto degli Abruzzi, Teramo, Italia) for selective growth of streptococci and enterococci; for blood cultures containing both streptococci/ enterococci and Gram-negative bacteria, $200 \mu \mathrm{L}$ of $2 \mu \mathrm{g} /$ $\mathrm{mL}$ colistin were added to $2 \mathrm{~mL}$ of Todd Hewitt broth to effectively inhibit the growth of Gram-negative bacteria; m-Staphylococcus Broth (Becton Dickinson and Company), containing $7.5 \% \mathrm{NaCl}$, for selective cultivation of staphylococci. The selectivity of this medium was enhanced by adding $300 \mu \mathrm{L}$ of a sterile solution of $\mathrm{NaCl}$ at increasing concentrations. The optimal concentration of $\mathrm{NaCl}$ was determined experimentally at $12 \%$, as allowing the growth of staphylococci while effectively inhibiting the growth of Gram-negative bacteria, streptococci and enterococci. Finally, HB\&L SABOURAUD (Alifax SpA, Polverara, $\mathrm{PD}$, Italy), was used for selective growth of yeast.

The different selective liquid media, dispensed in $2 \mathrm{~mL}$ aliquots, were inoculated with $200 \mu \mathrm{L}$ of bacterial suspension at $0.65 \mathrm{McFarland}$, and incubated at $37^{\circ} \mathrm{C}$ in the Alfred 60/AST instrument (Alifax), allowing a continuous monitoring of bacterial growth, until a turbidity of 0.5 McFarland was reached. Then, an aliquot of $1.5 \mathrm{~mL}$ of culture was transferred into a microcentrifuge tube, and centrifuged at 13,000 rpm for $2 \mathrm{~min}$; the supernatant was removed, and the pellet was used for microbial identification by MALDI-TOF MS as described above.

For identification of yeast, a protein extraction was carried out before MALDI-TOF MS analysis. To this purpose, the standard protocol provided by Bruker was slightly modified. The yeast pellet was resuspended in $75 \%$ ethanol, followed by centrifugation at $13,000 \mathrm{rpm}$ for $2 \mathrm{~min}$; the supernatant was discarded and the pellet was added $50 \mu \mathrm{L}$ of $70 \%$ formic acid and $50 \mu \mathrm{L}$ acetonitrile. After vortexing for $2 \mathrm{~min}$, the lysate was centrifuged at 13,000 rpm for $2 \mathrm{~min}$. The supernatant $(1 \mu \mathrm{L})$, containing the majority of yeast protein, was airdried and, after addition of the HCCA matrix, used for MALDI-TOF MS analysis as described.

An aliquot of each culture was plated onto TSH blood agar for $\mathrm{CFU} / \mathrm{mL}$ determination as described above.

\section{Abbreviations}

AST: Antimicrobial susceptibility testing; BC: Blood culture; BCID: Blood culture identification panel; CFU: Colony forming unit; HCCA: Alpha-cyano-4hydroxycinnamic acid; MALDI-TOF MS: Matrix-assisted laser desorption/ ionization time of flight mass spectrometry; $\mathrm{NaCl}$ : Sodium chloride; rpm: Revolutions per minute; TSH: Trypticase soy agar plus 5\% sheep blood

\section{Acknowledgements}

We thank Alifax SpA (Polverara, PD, Italy) for kindly providing part of the study material.

\section{Authors' contributions}

Conceived and designed the experiments: $\mathrm{AL}, \mathrm{AV}$. Performed the experiments: SC, CG. Analyzed the data: WF, AL. Wrote the manuscript: WF, SC. Critically revised the manuscript: AL, EG. All authors have read and approved the manuscript.

\section{Funding}

This research received no specific grant from any funding agency in the public, commercial, or not-for-profit sectors.

\section{Availability of data and materials}

The datasets used and/or analyzed during the current study are available from the corresponding author on reasonable request.

\section{Ethics approval and consent to participate}

This study was approved by the local ethical committee, Comitato Etico di Area Vasta Nord-Ovest, University of Pisa, and conducted in full accordance with the principles of the Declaration of Helsinki. Samples were taken as part of the standard patient care, anonymized by the clinical personnel, and used anonymously by the Research personnel. According to the local ethical committee, for this type of study, formal consent to participate was not required (Prot. N. 32634)

\section{Consent for publication}

Not applicable.

\section{Competing interests}

The authors declare that they have no competing interests.

Received: 28 August 2019 Accepted: 14 November 2019

Published online: 29 November 2019

\section{References}

1. Sogaard M, Norgaard M, Dethlefsen C, Schonheyder HC. Temporal changes in the incidence and 30-day mortality associated with bacteremia in hospitalized patients from 1992 through 2006: a population-based cohort study. Clin Infect Dis. 2011;52:61-9.

2. Bassetti M, Trecarichi EM, Mesini A, Spanu T, Giacobbe DR, Rossi M, et al. Risk factors and mortality of healthcare-associated and community-acquired Staphylococcus aureus bacteraemia. Clin Microbiol Infect. 2012;18(9):862-9.

3. Goto M, Al-Hasan MN. Overall burden of bloodstream infection and nosocomial bloodstream infection in North America and Europe. Clin Microbiol Infect. 2013;19(6):501-9.

4. Akova M. Epidemiology of antimicrobial resistance in bloodstream infections. Virulence. 2016;7(3):252-66.

5. Xu L, Sun X, Ma X. Systematic review and meta-analysis of mortality of patients infected with carbapenem-resistant Klebsiella pneumoniae. Ann Clin Microbiol Antimicrob. 2017;16(1):18. 
6. Bouza E, Burillo A, Muñoz P, Guinea J, Marín M, Rodríguez-Créixems M. Mixed bloodstream infections involving bacteria and Candida spp. J Antimicrob Chemother 2013;68(8):1881-8.

7. Royo-Cebrecos C, Gudiol C, Ardanuy C, Pomares H, Calvo M, Carratalà J. A fresh look at polymicrobial bloodstream infection in cancer patients. PLoS One. 2017:12(10):e0185768.

8. Espejo E, Andrés M, Borrallo R-M, Padilla E, Garcia-Restoy E, Bella F. Complex wounds working group. Bacteremia associated with pressure ulcers: a prospective cohort study. Eur J Clin Microbiol Infect Dis. 2018;37:969-75.

9. Ferreira L, Sanchez-Juanes F, Porras-Guerra I, Garcia-Garcia MI, Garcia-Sanchez JE, González-Buitrago JM, Muñoz-Bellido JL. Microorganisms direct identification from blood culture by matrix-assisted laser desorption/ionization time-of-flight mass spectrometry. Clin Microbiol Infect. 2011;17:546-51.

10. Huang AM, Newton D, Kunapuli A, Gandhi TN, Washer LL, Isip J, et al. Impact of rapid organism identification via matrix-assisted laser desorption/ ionization time-of-flight combined with antimicrobial stewardship team intervention in adult patients with bacteremia and candidemia. Clin Infect Dis. 2013;57:1237-45

11. Lévesque S, Dufresne PJ, Soualhine H, Domingo MC, Bekal S, Lefebvre B Tremblay C. A side by side comparison of Bruker Biotyper and VITEK MS: utility of MALDI-TOF MS technology for microorganism identification in a public health reference laboratory. PLoS One. 2015;10(12):e0144878.

12. Barnini S, Ghelardi E, Brucculeri V, Morici P, Lupetti A. Rapid and reliable identification of gram-negative bacteria and gram-positive cocci by deposition of bacteria harvested from blood cultures onto the MALDI-TOF plate. BMC Microbiol. 2015;15:124

13. Florio W, Morici P, Ghelardi E, Barnini S, Lupetti A. Recent advances in the microbiological diagnosis of bloodstream infections. Crit Rev Microbiol. 2018:44(3):351-70.

14. Lagace-Wiens PR, Adam HJ, Karlowsky JA, Nichol KA, Pang PF, Guenther J, et al. Identification of blood culture isolates directly from positive blood cultures by use of matrix-assisted laser desorption ionization-time of flight mass spectrometry and a commercial extraction system: analysis of performance, cost, and turnaround time. J Clin Microbiol. 2012;50:3324-8.

15. Martinez RM, Bauerle ER, Fang FC, Butler-Wu SM. Evaluation of three rapid diagnostic methods for direct identification of microorganisms in positive blood cultures. J Clin Microbiol. 2014;52:2521-9.

16. Chien J-Y, Lee T-F, Du S-H, Teng S-H, Liao C-H, Sheng W-H, et al. Applicability of an in-house saponin-based extraction method in Bruker Biotyper matrix-assisted laser desorption/ionization time-of-flight mass spectrometry system for identification of bacterial and fungal species in positively flagged blood cultures. Front Microbiol. 2016;7:1432.

17. Tian Y, Zheng B, Wang B, Lin Y, Li M. Rapid identification and multiple susceptibility testing of pathogens from positive-culture sterile body fluids by a combined MALDI-TOF mass spectrometry and Vitek susceptibility system. Front Microbiol. 2016;7:523.

18. Tanner H, Evans JT, Gossain S. Hussain A Evaluation of three sample preparation methods for the direct identification of bacteria in positive blood cultures by MALDI-TOF BMC Res Notes. 2017;10:48.

19. Zhou M, Yang Q, Kudinha T, Sun L, Zhang R, Liu C, et al. An improved inhouse MALDI-TOF MS protocol for direct cost-effective identification of pathogens from blood cultures. Front Microbiol. 2017;8:1824.

20. Lee AWT, Lam JKS, Lam RKW, Ng WH, Lee ENL, Lee VTY, et al. Comprehensive evaluation of the MBT STAR-BL module for simultaneous bacterial identification and $\beta$-lactamase-mediated resistance detection in gram-negative rods from cultured isolates and positive blood cultures. Front Microbiol. 2018;9:334.

21. Mancini N, Infurnari L, Ghidoli N, Valzano G, Clementi N, Burioni R, Clementi M. Potential impact of a microarray-based nucleic acid assay for rapid detection of gram-negative bacteria and resistance markers in positive blood cultures. J Clin Microbiol. 2014;52(4):1242-5.

22. Ledeboer NA, Lopansri BK, Dhiman N, Cavagnolo R, Carroll KC, Granato P, et al. Identification of gram-negative bacteria and genetic resistance determinants from positive blood culture broths by use of the Verigene gram-negative blood culture multiplex microarray-based molecular assay. J Clin Microbiol. 2015;53(8):2460-72.

23. Claeys KC, Heil EL, Pogue JM, Lephart PR, Johnson JK. The Verigene dilemma: gram-negative polymicrobial bloodstream infections and clinical decision making. Diagn Microbiol Infect Dis. 2018;91:144-6.

24. Kohlmann R, Hoffmann A, Geis G, Gatermann S. MALDI-TOF mass spectrometry following short incubation on a solid medium is a valuable tool for rapid pathogen identification from positive blood cultures. Int $J$ Med Microbiol. 2015;305:469-79.

25. Ferroni A, Suarez S, Beretti JL, Dauphin B, Bille E, Meyer J, et al. Real-time identification of bacteria and Candida species in positive blood culture broths by matrix-assisted laser desorption ionization-time of flight mass spectrometry. J Clin Microbiol. 2010;48:1542-8.

26. Scohy A, Noël A, Boeras A, Brassinne L, Laurent T, Rodriguez-Villalobos $H$, Verroken A. Evaluation of the Bruker ${ }^{\ominus}$ MBT Sepsityper IVD module for the identification of polymicrobial blood cultures with MALDI-TOF MS. Eur J Clin Microbiol Infect Dis. 2018;37:2145-52

27. Descours G, Desmurs L, Hoang TLT, Ibranosyan M, Baume M, Ranc A-G, et al. Evaluation of the accelerate Pheno ${ }^{\mathrm{TM}}$ system for rapid identification and antimicrobial susceptibility testing of gram-negative bacteria in bloodstream infections. Eur J Clin Microbiol Infect Dis. 2018;37:1573-83.

28. Ward C, Stocker K, Begum J, Wade P, Ebrahimsa U, Goldenberg SD. Performance evaluation of the Verigene ${ }^{\oplus}$ (Nanosphere) and FilmArray ${ }^{\ominus}$ (BioFire $\left.{ }^{\oplus}\right)$ molecular assays for identification of causative organisms in bacterial bloodstream infections. Eur J Clin Microbiol Infect Dis. 2016;34(3):487-96.

29. Beal SG, Ciurca J, Smith G, John J, Lee F, Doern CD, Gander RM. Evaluation of the Nanosphere Verigene gram-positive blood culture assay with the VersaTREK blood culture system and assessment of possible impact on selected patients. J Clin Microbiol. 2013:51:3988-92.

30. Fiori B, D'Inzeo T, Giaquinto A, Menchinelli G, Liotti FM, de Maio F, et al. Optimized use of the MALDI BioTyper system and the FilmArray BCID panel for direct identification of microbial pathogens from positive blood cultures. J Clin Microbiol. 2016:54(3):576-84.

31. Idelevich EA, Grunewald CM, Wüllenweber J, Becker K. Rapid identification and susceptibility testing of Candida spp. from positive blood cultures by combination of direct MALDI-TOF mass spectrometry and direct inoculation of Vitek 2. PLoS One. 2014;9:e114834.

32. Morgenthaler NG, Kostrzewa M. Rapid identification of pathogens in positive blood culture of patients with sepsis: review and meta-analysis of the performance of the Sepsityper kit. Int J Microbiol. 2015;827416.

33. Barnini S, Brucculeri V, Morici P, Ghelardi E, Florio W, Lupetti A. A new rapid method for direct antimicrobial susceptibility testing of bacteria from positive blood cultures. BMC Microbiol. 2016;16(1):185.

34. Vecchione A, Florio W, Celandroni F, Barnini S, Lupetti A, Ghelardi E. A rapid procedure for identification and antifungal susceptibility testing of yeasts from positive blood cultures. Front Microbiol. 2018;9:2400.

35. Vrioni G, Tsiamis C, Oikonomidis G, Theodoridou K, Kapsimali V, Tsakris A. MALDI-TOF mass spectrometry technology for detecting biomarkers of antimicrobial resistance: current achievements and future perspectives. Ann Transl Med. 2018;6(12):240.

36. Florio W, Tavanti A, Ghelardi E, Lupetti A. MALDI-TOF MS applications to the detection of antifungal resistance: state of the art and future perspectives. Front Microbiol. 2018;9:2577.

37. Burckhardt I, Zimmermann S. Susceptibility testing of bacteria using MALDITOF mass spectrometry. Front Microbiol. 2018;9:1744.

\section{Publisher's Note}

Springer Nature remains neutral with regard to jurisdictional claims in published maps and institutional affiliations.

Ready to submit your research? Choose BMC and benefit from:

- fast, convenient online submission

- thorough peer review by experienced researchers in your field

- rapid publication on acceptance

- support for research data, including large and complex data types

- gold Open Access which fosters wider collaboration and increased citations

- maximum visibility for your research: over $100 \mathrm{M}$ website views per year

At $\mathrm{BMC}$, research is always in progress.

Learn more biomedcentral.com/submission 